\title{
Stent screw-assisted internal fixation (SAIF): clinical report of a novel approach to stabilizing and internally fixating vertebrae destroyed by malignancy
}

\author{
Alessandro Cianfoni, MD, ${ }^{1,2}$ Daniela Distefano, MD, ${ }^{1}$ Pietro Scarone, MD, ${ }^{3}$ \\ Gianfranco A. Pesce, MD, ${ }^{4}$ Vittoria Espeli, MD, ${ }^{5}$ Luigi La Barbera, PhD, ${ }^{6-8}$ Tomaso Villa, PhD, ${ }^{6}$ \\ Michael Reinert, MD, ${ }^{3,9}$ Giuseppe Bonaldi, MD, ${ }^{10}$ and Joshua A. Hirsch, MD ${ }^{11}$ \\ ${ }^{1}$ Department of Neuroradiology, Neurocenter of Southern Switzerland, Ospedale Regionale di Lugano; '2Department of \\ Interventional and Diagnostic Neuroradiology, Inselspital University Hospital of Bern; ${ }^{3}$ Department of Neurosurgery, Neurocenter \\ of Southern Switzerland, Ospedale Regionale di Lugano; Departments of ${ }^{4}$ Radiation Oncology and ${ }^{5}$ Medical Oncology, Oncology \\ Institute of Southern Switzerland, Ospedale Regionale di Bellinzona e Valli, San Giovanni, Bellinzona, Switzerland; ${ }^{6}$ Laboratory \\ of Biological Structure Mechanics, Department of Chemistry, Materials and Chemical Engineering "Giulio Natta", Politecnico di \\ Milano, Milan, Italy; ' ${ }^{7}$ epartment of Mechanical Engineering, Polytechnique Montréal; ${ }^{8}$ Sainte-Justine Clinical Hospital Center, \\ Montréal, Quebec, Canada; ${ }^{9}$ Department of Neurosurgery, Inselspital University Hospital of Bern, Switzerland; ${ }^{10}$ Department \\ of Neurosurgery, Igea Clinic, Milan, Italy; and ${ }^{11}$ Department of Radiology, Massachusetts General Hospital, Harvard Medical \\ School, Boston, Massachusetts
}

OBJECTIVE Severe lytic cancerous lesions of the spine are associated with significant morbidity and treatment challenges. Stabilization and restoration of the axial load capability of the vertebral body (VB) are important to prevent or arrest vertebral collapse. Percutaneous stent screw-assisted internal fixation (SAIF), which anchors a VB stent/cement complex with pedicular screws to the posterior vertebral elements, is a minimally invasive, image-guided, $360^{\circ}$ internal fixation technique that can be utilized in this patient cohort. The purpose of this study was to assess the feasibility, safety, and stabilization efficacy of VB reconstruction via the SAIF technique in a cohort of patients with extensive lytic vertebral lesions, who were considered to have an unstable or potentially unstable spine according to the Spinal Instability Neoplastic Score (SINS).

METHODS This study was a retrospective assessment of a prospectively maintained database of a consecutive series of patients with neoplastic extensive extracompartmental osteolysis (Tomita type 4-6) of the VB treated with the SAIF technique. VB reconstruction was assessed on postprocedure plain radiographs and CT by two independent raters. Technical and clinical complications were recorded. Clinical and imaging follow-ups were assessed.

RESULTS Thirty-five patients with extensive osteolytic metastatic lesions of the VB underwent 36 SAIF procedures. SAIF was performed as a stand-alone procedure in $31 / 36$ cases and was associated with posterior surgical fixation in $5 / 36$ (4/5 with decompressive laminectomy). In 1 case an epidural cement leak required surgical decompression. VB reconstruction was categorized as satisfactory (excellent or good rating) by the two raters in 34/36 cases (94.5\%) with an interrater reliability of $94.4 \%$ (Cohen's kappa of 0.8 ). Follow-up, ranging from 1 to 30 months, was available for 30/36 levels. Long-term follow-up (6-30 months, mean 11.5 months) was available for $16 / 36$ levels. Stability during follow-up was noted in 29/30 cases.

CONCLUSIONS SAIF provides $360^{\circ}$ nonfusion internal fixation that stabilizes the VB in patients with extensive lytic lesions that would otherwise be challenging to treat.

https://thejns.org/doi/abs/10.3171/2019.9.SPINE19711

KEYWORDS extreme osteolysis; pathological fracture; vertebral body stents; pedicular screws; stabilization; internal fixation; stent screw-assisted internal fixation; SAIF; oncology

ABBREVIATIONS EO = extreme osteolysis; ESCC = epidural spinal cord compression; PMMA = polymethyl methacrylate; SAIF = stent screw-assisted internal fixation; SINS = Spinal Instability Neoplastic Score; VA = vertebral augmentation; VB = vertebral body; VBS = VB stent.

SUBMITTED July 1, 2019. ACCEPTED September 30, 2019.

INCLUDE WHEN CITING Published online December 20, 2019; DOI: 10.3171/2019.9.SPINE19711. 
$\mathrm{E}$ XTENSIVE neoplastic osteolysis of the vertebral body (VB) can significantly impact the load-bearing capacity of the spine,,$^{38}$ leading to instability, fractures, and potential neurological deficit. ${ }^{34}$ While percutaneous augmentation procedures have traditionally focused on pain palliation, mechanical stabilization is an independent surgical indication.

Surgical treatment is effective at restoring the mechanical stability of the anterior and posterior columns, ${ }^{39}$ but instrumented fusion may not always be appropriate in cases of multilevel metastatic involvement or in patients with poor bone quality. Moreover, corpectomy and grafting, which reinforce the anterior column, are invasive and can lead to significant morbidity, especially in medically fragile patients. ${ }^{6,22,25,44}$

Vertebral augmentation (VA), performed with vertebroplasty or balloon kyphoplasty, is a minimally invasive option that reinforces the anterior column, ${ }^{4}$ but when osteolysis causes extensive destruction of the cortical boundaries of the VB, there is risk of cement leakage, possibly resulting in neural compression or insufficient augmentation of the VB.,21 In the present study, we address the stabilization of such lesions, characterized by "extreme osteolysis" (EO) with wide circumferential discontinuity of the cortical boundaries of the VB, corresponding to extracompartmental type 4-6 lytic lesions in the Tomita classification (Fig. 1). ${ }^{41}$

VB stents (VBSs) are barrel-shaped, balloon-expandable metallic cages that can be used to reduce the vertebral fracture, ${ }^{15,43}$ reconstruct the VB, and aid in cement augmentation of EO cases. ${ }^{9}$ Nevertheless, in a VB with extensive cortical dehiscence, there is risk of VBS/cement mobilization before new bone apposition occurs. ${ }^{9}$

The recently described percutaneous stent screw-assisted internal fixation (SAIF), ${ }^{8}$ whereby the VBS/cement complex is anchored with pedicular screws to the posterior vertebral elements, is a minimally invasive, image-guided, $360^{\circ}$ internal fixation technique. It can be performed as a stand-alone procedure or in combination with a posterior surgical approach to stabilize the anterior column. We hypothesized that SAIF can be successfully employed in EO as a means of VB reconstruction and restoration of axial load capability with the aim of obviating the need for a more invasive corpectomy and grafting. The purpose of this study was to assess the feasibility, safety, and stabilization efficacy of VB reconstruction via the SAIF technique in a cohort of patients with $\mathrm{EO}$ of the $\mathrm{VB}$, who were considered to have an unstable or potentially unstable spine according to the Spinal Instability Neoplastic Score (SINS). ${ }^{18}$

\section{Methods \\ Patients}

This is a retrospective analysis of a single-center, prospectively maintained database of a consecutive series of patients with neoplastic EO of one or more thoracolumbar VBs treated with SAIF between May 2015 and September 2018. The study was authorized by the Ethics Committee of Canton Ticino.

All patients underwent preprocedure $\mathrm{CT}$ and gadolinium-enhanced MRI of the spine at the target level to define the extent of lytic lesions, the degree of vertebral collapse ( $\leq$ or $>50 \%$ ), and the presence of an associated epidural mass defined as epidural spinal cord compression (ESCC) grade $^{5}$ between $1 \mathrm{~b}$ and 3 , inclusive. All target lesions were deemed to be unstable or potentially unstable according to the SINS. ${ }^{18}$

An individualized clinical decision on the treatment plan for each patient was reached by a multidisciplinary spine tumor board composed of medical and radiation oncologists, spine surgeons, and neuroradiologists, who defined indications for and the timing of invasive treatment, radiation, and chemotherapy. Informed consent was obtained for all procedures.

\section{SAIF Procedure}

SAIF procedural details have been previously described. ${ }^{8}$ Briefly, all procedures were performed percutaneously while the patient was under general anesthesia, with the aid of biplane fluoroscopic guidance. When deemed necessary, cavity creation was performed utilizing curettage and vacuum suction (Q-VAC technique). ${ }^{35}$ Following transpedicular implant of the VBS(s) and placement of uni- or bilateral pedicular screws, cement augmentation was performed through the screw(s) with high-viscosity polymethyl methacrylate (PMMA; VertaPlex HV, Stryker) under real-time fluoroscopy.

In cases of multilevel lytic lesions, when deemed appropriate, additional vertebrae at adjacent or distant levels were treated with cement-only VA during the same session. Patients undergoing a stand-alone SAIF procedure were allowed to stand and walk without spinal braces as early as 3 hours after the procedure. Most SAIF procedures were performed in a day surgery setting.

\section{Assessment of VB Reconstruction, Complications, and Follow-Up}

VB reconstruction was assessed with postprocedure plain radiographs and CT scans. CT data sets were reconstructed utilizing a bone algorithm with 3 - and $10-\mathrm{mm}$ thick maximum intensity projection (MIP) images in three orthogonal planes. A neuroradiologist (A.C.) and a neurosurgeon (P.S.) independently reviewed the postprocedure images to assess the technical results, assigning a VB reconstruction "score." We applied a previously published, qualitative 4-grade scale (poor, fair, good, excellent), ${ }^{9}$ based on the overall assessment of correct placement and expansion of the implants, cement filling of the lytic cavities, and restoration of the VB height. A poor grade was characterized as a failure to achieve sufficient augmentation of the anterior column, whereas an excellent grade indicated stent expansion and cement distribution filling the lytic lesion and reconstructing the destroyed portion of the VB, satisfactory height restoration, and correct screw positioning.

Intraprocedural complications, including potentially significant cement leaks, and misplacement of the screws were recorded.

At 1 month after treatment, patients were followed up with clinical examination and upright spine radiographs. Patients underwent routine oncological clinical and imag- 


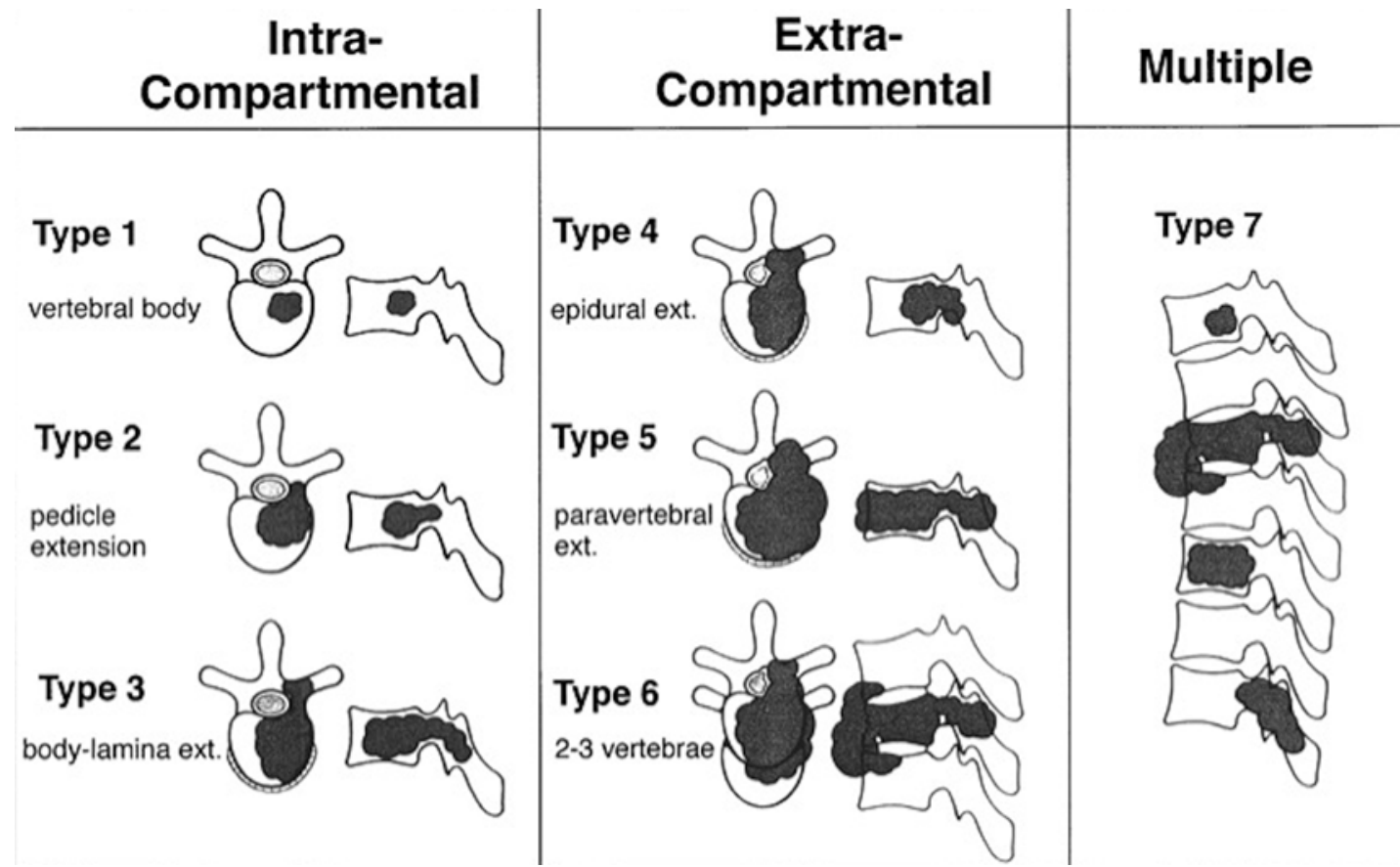

FIG. 1. Schematic of Tomita's classification of spinal metastases. Lesions in the present study featured extensive osteolysis of the VB with wide cortical discontinuity, defined as "extreme osteolysis," corresponding to extracompartmental type 4-6 lesions in the Tomita classification. Reprinted with permission from Tomita K, Kawahara N, Kobayashi T, Yoshida A, Murakami H, Akamaru T: Surgical strategy for spinal metastases. Spine (Phila Pa 1976) 26 (3):298-306, 2001. https://journals.Iww.com/spinejournal/.

ing follow-up with CT, PET-CT, MRI, or plain radiography, as clinically indicated. Those clinical charts and imaging studies were reviewed as part of this study. Imaging follow-up data were evaluated to assess the recurrence of vertebral collapse, new or worsening spinal deformity of the treated segment, mobilization of the implants, signs of subsidence, new vertebral fractures at adjacent levels, and local tumor progression. Patients were referred to the multidisciplinary spine tumor board for any recurrent or new spinal events.

\section{Statistical Analysis}

Analyses were conducted using SPSS version 20.0.0 (IBM Corp.). Descriptive statistics for clinical and demographic data were expressed as the mean or median and range. VB reconstruction results were judged according to the following scale: poor, fair, good, or excellent. Excellent and good ratings were considered as satisfactory results. ${ }^{9}$ Cohen's kappa coefficient $(\kappa)$ was used to assess the proportion of agreement of the two independent raters beyond that expected by chance, ${ }^{11}$ and the classification by Landis and $\mathrm{Koch}^{32}$ was used to define the agreement level: poor, $<0.00$; slight, $0.00-0.20$; fair, 0.21-0.40; moderate, $0.41-$ 0.60 ; substantial, $0.61-0.80$; or almost perfect, $0.81-1.00$.

\section{Results}

In 35 patients, $36 \mathrm{SAIF}$ procedures were performed to treat 36 levels with EO. A summary of patient characteristics and features of the lytic lesions is provided in Table 1 , whereas technical results and periprocedural complications are summarized in Table 2.

\section{Technical Results}

SAIF procedures were performed as a stand-alone intervention in 31/36 cases, in conjunction with percutaneous posterior surgical fixation in 1/36 cases, and after decompressive laminectomy and posterior surgical fixation in 4/36 cases. Bilateral VBS implants were utilized in 33/36 levels (92\%), and a unilateral VBS implant was used in $3 / 36$ levels $(8 \%)$. Bilateral screws were utilized in $18 / 36$ levels (50\%), and unilateral screws were used in 18/36 levels $(50 \%)$. Cavity creation in the VB with Q-VAC ${ }^{35}$ was performed in 29/36 levels.

During the same procedure, cement-only VA was performed at adjacent or distant vertebral levels (i.e., affected by lytic lesions, but not defined as EO) in 22/35 patients, at a total of 61 levels.

\section{VB Reconstruction}

VB reconstruction scores assigned by the two raters were, respectively, excellent at 27/36 (75\%) and 28/36 (78\%) levels, good at 7/36 (19.4\%) and 6/36 (16.7\%), fair at $2 / 36(5.6 \%)$ and $2 / 36(5.6 \%)$, and poor at none of the treated levels, with satisfactory results (excellent or good rating) in 34/36 cases $(94.5 \%)$ for both raters. The interrater reliability was $94.4 \%$ with a Cohen's kappa of 0.8 indicating strong interrater reliability.

\section{Procedural and Periprocedural Safety}

An extravertebral cement leak was visible at 12/36 levels $(33 \%)$ on postprocedure CT, with an epidural or a foraminal location in 7/36 levels (19\%). In 1 case the epidural cement leak caused the postprocedural onset of an L2 
TABLE 1. Characteristics of the study population and features of lytic lesions

\begin{tabular}{|c|c|}
\hline Variable & Value \\
\hline Total no. of patients & 35 \\
\hline Sex: M/F & $21 / 14$ \\
\hline Mean age in yrs (range) & $69.9(43-87)$ \\
\hline \multicolumn{2}{|l|}{ ECOG score } \\
\hline Mean & 1.62 \\
\hline Median & 1 \\
\hline Range & $0-4$ \\
\hline Treated levels & 36 \\
\hline Thoracic & $16 / 36(44.4 \%)$ \\
\hline Lumbar & $20 / 36(55.6 \%)$ \\
\hline \multicolumn{2}{|l|}{ Primary cancer, no. of levels (\%) } \\
\hline Solid tumor metastases & $31(86.1)$ \\
\hline Multiple myeloma/plasmocytoma & $5(13.9)$ \\
\hline Epidural mass, no. of levels (\%) & $23 / 36(63.9)$ \\
\hline \multicolumn{2}{|l|}{ ESCC grade, no. of levels } \\
\hline 0 & 8 \\
\hline $1 \mathrm{a}$ & 5 \\
\hline $1 b$ & 9 \\
\hline 1c & 5 \\
\hline 2 & 5 \\
\hline 3 & 4 \\
\hline \multicolumn{2}{|l|}{ VB collapse, no. of levels ( $\%$ ) } \\
\hline$\leq 50 \%$ & $14(38.9)$ \\
\hline$>50 \%$ & $22(61.1)$ \\
\hline \multicolumn{2}{|l|}{ SINS } \\
\hline Mean & 10.8 \\
\hline Median & 11 \\
\hline Range & $7-14$ \\
\hline
\end{tabular}

ECOG = Eastern Cooperative Oncology Group; ESCC $=$ epidural spinal cord compression; SINS = Spinal Instability Neoplastic Score.

sensory and motor deficit necessitating emergent L2 decompressive hemilaminectomy, with complete resolution of symptoms. In another case a small epidural leak caused transient, self-resolving L5 radicular pain. The remaining cement leaks were asymptomatic. No other patients experienced worsening of their neurological status after the procedure. One patient with a preexisting neurological deficit due to spinal cord compression underwent SAIF associated with laminectomy and posterior instrumentation and did not experience worsening of his neurological condition. One patient who had undergone SAIF combined with laminectomy and surgical stabilization subsequently developed a pulmonary embolism on day 1 postprocedure, with no evidence of PMMA emboli, and died on day 2. No other periprocedural clinical complications occurred.

\section{Follow-Up Results and Nonneurological Complications}

A summary of follow-up results is provided in Table 3. Postprocedure clinical and imaging follow-up was available at 1 month for 30/36 treated levels, at 3 months for $24 / 36$ levels, and at 6 months or later (range 6-30 months, mean 11.5 months) for $16 / 36$ levels.

In 1 patient treated with unilateral-screw SAIF, the
TABLE 2. Summary of technical results and periprocedural complications

\begin{tabular}{|c|c|}
\hline Variable & Value \\
\hline \multicolumn{2}{|l|}{ Procedure, no. of levels (\%) } \\
\hline Stand-alone SAIF & $31 / 36(86.1)$ \\
\hline SAIF + pPF & $1 / 36(2.8)$ \\
\hline SAIF + L-PF & $4 / 36(11.1)$ \\
\hline \multicolumn{2}{|l|}{ VBS, no. of levels (\%) } \\
\hline Bilat & $33 / 36(91.7)$ \\
\hline Unilat & $3 / 36(8.3)$ \\
\hline \multicolumn{2}{|l|}{ Screws, no. of levels (\%) } \\
\hline Bilat & $18 / 36(50.0)$ \\
\hline Unilat & $18 / 36(50.0)$ \\
\hline Cavity creation, no. of levels (\%) & $29 / 36(80.6)$ \\
\hline \multicolumn{2}{|l|}{ VA (cement only) } \\
\hline No. of patients & $22 / 35$ \\
\hline No. of levels & 61 \\
\hline \multicolumn{2}{|l|}{ VB reconstruction scale, no. of levels (\%) } \\
\hline \multicolumn{2}{|l|}{ Reader 1} \\
\hline Excellent & $27 / 36(75.0)$ \\
\hline Good & $7 / 36(19.4)$ \\
\hline Fair & $2 / 36(5.6)$ \\
\hline Poor & $0 / 36(0)$ \\
\hline \multicolumn{2}{|l|}{ Reader 2} \\
\hline Excellent & $28 / 36(77.8)$ \\
\hline Good & $6 / 36(16.7)$ \\
\hline Fair & $2 / 36(5.6)$ \\
\hline Poor & $0 / 36(0)$ \\
\hline Cement leak, no. of levels (\%) & $12 / 36(33.3)$ \\
\hline Epidural-foraminal space & $7 / 36(19.4)$ \\
\hline \multicolumn{2}{|l|}{ Periprocedural complication, no. of levels } \\
\hline $\begin{array}{l}\text { Cement leak requiring surgery w/ no permanent } \\
\text { neurological deficit }\end{array}$ & $1 / 36$ \\
\hline Self-limiting neurological symptom & $1 / 36$ \\
\hline Death (day 2, PE) & $1 / 36$ \\
\hline
\end{tabular}

$\mathrm{L}-\mathrm{PF}=$ decompressive laminectomy and posterior fixation; $\mathrm{PE}=$ pulmonary embolism; pPF = percutaneous posterior fixation.

nonanchored VBS mobilized dorsally, toward the neuroforamen, 1 month postprocedure in the context of an unrecognized preexisting vertebral osteomyelitis. This required surgical removal, curettage, and stabilization. Implant stability was otherwise observed in all remaining cases until the last available follow-up. Mild subsidence of the treated VB around the VBS/cement complex was observed during follow-up in 5/30 levels (17\%) with available follow-up, without the onset of new symptoms. Intervertebral osseous fusion was noted in 4/16 levels (25\%) with long-term follow-up ( $\geq 6$ months). Local disease progression was observed in $2 / 30$ levels $(7 \%)$ at 3 and 5 months postprocedure, respectively, not affecting local spinal stability. No new fractures were noted at adjacent levels at the follow-up imaging.

\section{Discussion}

In this series of patients with EO and an SINS indicating potentially or frankly unstable lesions, the SAIF tech- 
TABLE 3. Summary of follow-up results

\begin{tabular}{ll}
\hline \multicolumn{1}{c}{ Variable } & No. of Levels $(\%)$ \\
\hline Follow-up, no. of levels & \\
1 mo & $30 / 36$ \\
3 mos & $24 / 36$ \\
$\geq 6$ mos $^{*}$ & $16 / 36$ \\
\hline VBS mobilization (non-screw-anchored VBS) & $1 / 30 \dagger$ \\
\hline Asymptomatic subsidence & $5 / 30 \dagger(17)$ \\
\hline Local progression & $2 / 30 \dagger(7)$ \\
\hline Intervertebral fusion & $4 / 16 \ddagger(25)$ \\
\hline
\end{tabular}

* Mean follow-up 11.5 months (range 6-30 months).

† Levels with available follow-up (range 1-30 months).

$\ddagger$ Levels with available late follow-up (range 6-30 months).

nique proved to be a feasible and safe minimally invasive procedure for VB reconstruction and stabilization, with durable results at follow-up. These clinical results seem to confirm recently published biomechanical data on a finite-element analysis (FEM) of the SAIF technique applied to a vertebra affected by EO.$^{31}$ SAIF was effective in recovering the axial stiffness and reducing the strains of the treated vertebra in the tested scenario. In contrast, a short posterior fixation was unable to significantly restore stiffness and stability. Posterior fixation combined with SAIF showed only minor additional effects as compared to those obtained with SAIF alone. These biomechanical results clearly underscore the importance of anterior column support during stabilization of osteolytic lesions of the VB.

Vertebroplasty and/or balloon kyphoplasty are widely used for pain palliation in cancer-related vertebral compression fractures, ${ }^{19,24,26}$ but when stability is a concern, posterior spinal instrumentation is warranted. ${ }^{33}$ Standalone VA is relatively contraindicated in the presence of vertebra plana, osseous comminution, posterior wall dehiscence, or wide cortical erosions. In fact, these were exclusion criteria in a prospective randomized controlled trial comparing kyphoplasty to nonsurgical management in cancer patients with vertebral fractures. ${ }^{1,4}$ While VA is sometimes done in these situations for pain palliation, such procedures do not address the instability.

VBS augmentation has been reported as a viable option to reconstruct the anterior column in $\mathrm{EO},{ }^{9}$ but when the VB's cortical boundaries are widely dehiscent, there is risk of VBS mobilization. ${ }^{9,28}$ Misplaced or dislocated stents outside the VB can cause serious complications such as injury to the nerve roots, spinal cord, lumbar plexus, great vessels, pleura, or mediastinal organs. ${ }^{40}$ For these reasons, at the institution at which this study was undertaken, the patients with EO would have been treated with anterior column reconstructive surgery combined with posterior instrumentation. Percutaneous image-guided screw fixation is an emerging procedure for osteosynthesis using cannulated cement-augmented screws, demonstrating promising results in the fixation of osteolytic bone metastasis in the spine and other skeletal locations. 7,13,14,20,30,36

The patients considered to be eligible for SAIF are medically fragile, which often limits the indication for corpectomy, anterior stabilization, and open posterior instrumentation. Beyond that, poor bone quality due to multilevel metastatic involvement and/or osteoporosis represents a condition predisposing to instrumentation failure.

This is the first reported series of patients with EO spinal lesions treated with the SAIF technique ${ }^{8}$ in which screws were used in combination with VBSs in EO spinal lesions with the intent to obtain $360^{\circ}$ nonfusion internal VB fixation. A VBS was used to restore VB height, scaffold the VB, help cement containment, and reinforce the anterior column extensively destroyed by the lytic lesion. The addition of the screws guaranteed anchoring of the VBS/PMMA implant to the generally intact posterior elements, bridging the middle column and preventing their mobilization. The combined use of VBS, PMMA, and pedicular screws can represent a VB prosthesis in these extensively destroyed metastatic lesions and can be used as a stand-alone technique or combined with posterior instrumentation and/or decompressive laminectomy or separation surgery, potentially obviating the need for corpectomy and grafting.

In this series, all patients were evaluated by multiple specialists in the setting of a multidisciplinary spine tumor board with extensive experience in the treatment of oncological disease. After that consultation, when deemed appropriate and if reconstructive anterior column surgery was contraindicated, SAIF was performed as a stand-alone procedure (Figs. 2-4) or was combined with a posterior surgical approach for external fixation (Fig. 5) and decompressive laminectomy. Even in this challenging cohort, characterized by higher SINSs (12-14) and higher ESCC grades (2-3), anterior and posterior stabilization was performed, avoiding more invasive surgical reconstruction of the anterior column and meaningful morbidity risks. ${ }^{6,16} \mathrm{In}$ this study, the SAIF group was not compared to a control group, as the patients in this series were in a very challenging clinical position with other clinical conditions and severe lesions of the VB that posed contraindications to standard augmentation procedures, making a more invasive anterior column surgery less palatable. ${ }^{2}$

\section{SAIF Technique}

SAIF was preferentially performed with bilateral VBS implants to offer more satisfactory VB reconstruction results. ${ }^{9}$ Ideally, the SAIF technique should be performed with bilateral screw fixation (Figs. 2 and 4), but when EO involved one of the two pedicles, a unilateral screw was inserted through the intact pedicle to ensure osseous anchoring to the VBS/cement complex (Figs. 3 and 5). Especially in these cases, the VBSs should be positioned converging toward the midline of the VB so that, upon expansion, the two stents could have a "kissing configuration," favoring the creation of a PMMA bridge between the two VBSs. The screw anchor and cement bridge ensure stability of the construct. In 3 cases, given the presence of mixed lytic and sclerotic components in the VB, a unilateral VBS was implanted in the lytic area.

The injected PMMA volume varied according to the size of the lytic lesions, trabecular compliance, stent expansion, and cement distribution. The goal is to obtain optimal cement filling and interdigitation in the nonlytic 

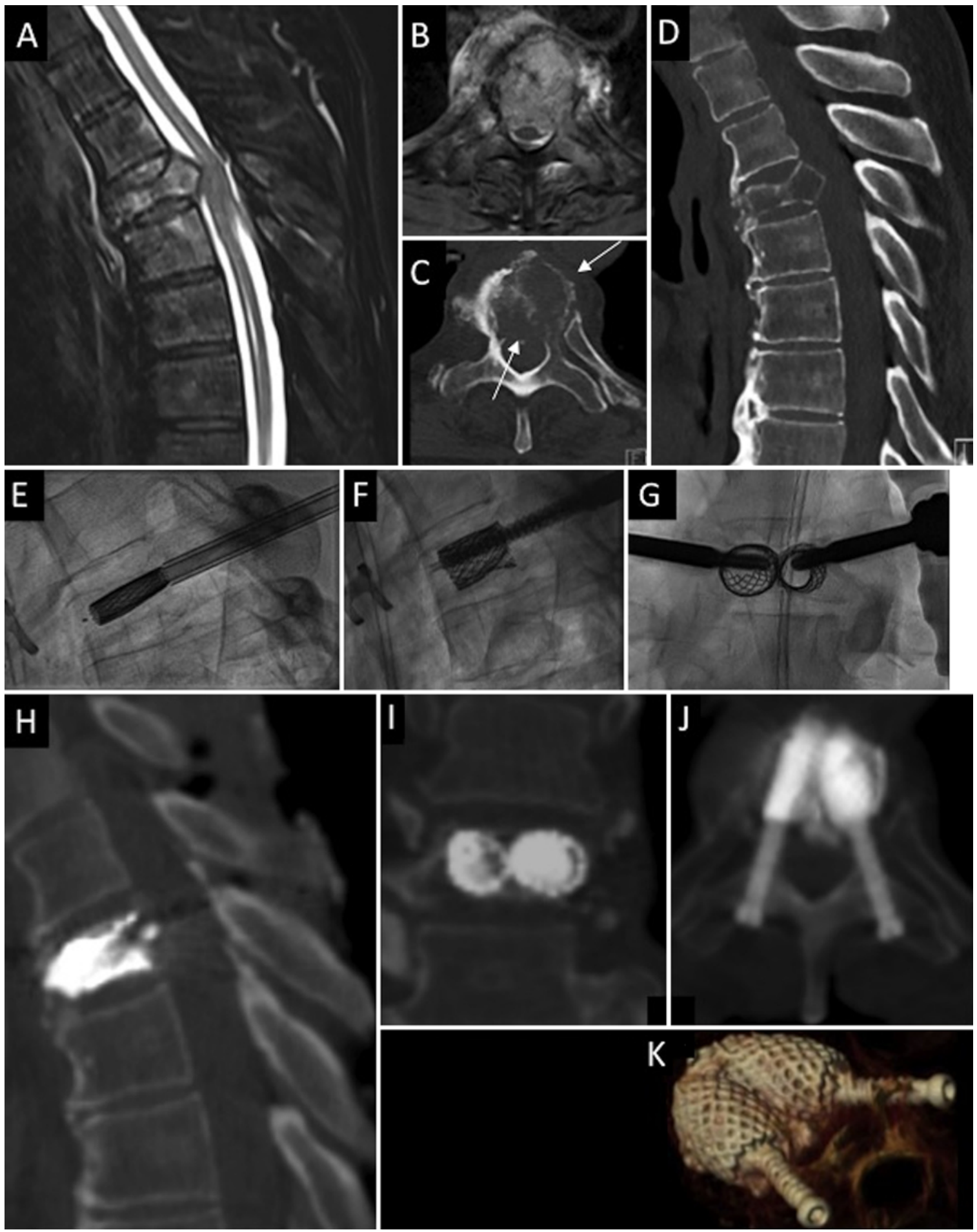

FIG. 2. Images obtained in the case of a lung cancer metastatic T4 fracture in a neurologically intact 72-year-old patient. Sagittal (A) and axial (B) fat-sat T2-weighted MRI showed VB collapse with central canal involvement and spinal cord compression, while CT (C-D) showed extensive osteolysis of trabecular and cortical VB components, namely posterior wall and left anterolateral wall dehiscence (arrows in C). The SAIF technique was performed with transpedicular stent placements (E) and expansion, followed by screw insertion $(\mathbf{F}-\mathbf{G})$ and cement augmentation. Postprocedure $\mathrm{CT}(\mathbf{H}-\mathbf{K})$ showed fracture reduction, posterior wall retropulsion correction due to ligamentotaxis, and the stents/cement complex anchored by screws to the posterior elements, acting as a VB prosthesis. Figure is available in color online only. 

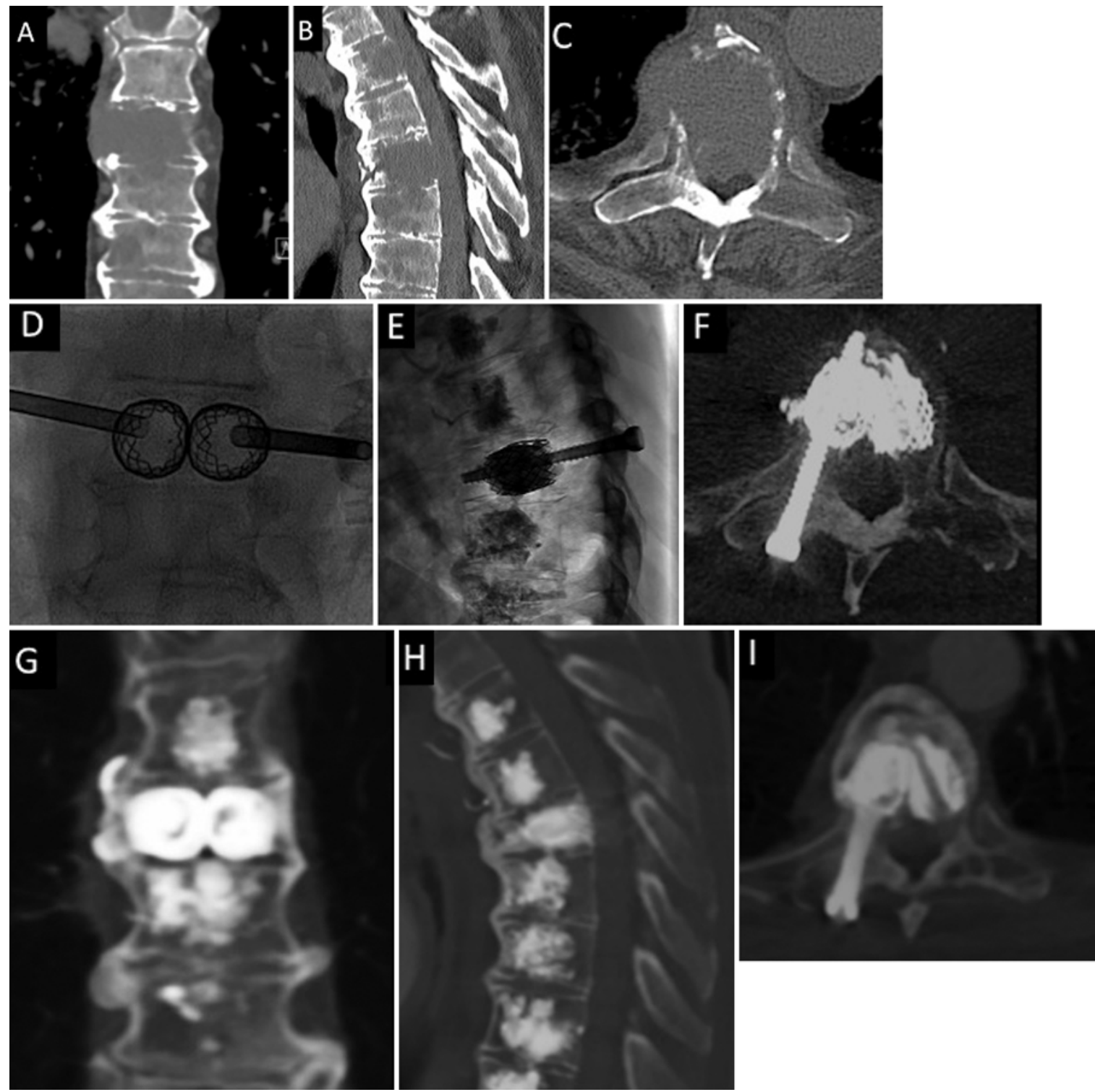

FIG. 3. Images obtained in a 67-year-old patient with multiple myeloma and multilevel spine osteolysis. CT studies (A-C) showed EO of T7 with largely discontinuous VB cortical boundaries and a situation of impending collapse. The SAIF technique with bilateral stents and a unilateral screw was performed at T7 (D-F), along with cement-only VA at multiple other levels weakened by nonextreme lytic lesions. Follow-up CT at 24 months postprocedure (G-I) showed osseous re-apposition in formerly lytic lesions, embedding of the stents/cement complex, and intervertebral fusion suggesting stability.

adjacent trabecular bone whenever possible. Techniques utilized to limit PMMA leakage and/or displacement of the soft tissue include the creation of a cavity, ${ }^{8,10,35}$ the use of high-viscosity PMMA, ${ }^{3,10}$ the containment effect of the VBS mesh, ${ }^{9}$ and real-time fluoroscopic control during cement injection. These approaches were considered particularly relevant, as these patients had highly destructive lesions typically with a dehiscent posterior wall and the frequent coexistence of an epidural mass.

There were 22/36 levels with a high degree of vertebral collapse and 23/36 levels with an epidural mass on preprocedure MRI. Although MRI postprocedure was not routinely performed to assess soft-tissue encroachment of the central canal, there was no worsening of neurological status postprocedure. We hypothesize that cavity creation and fracture reduction, with reexpansion of the collapsed VB in a craniocaudal direction, may have had a role in preventing clinically significant soft-tissue migration in the central canal. All screws were positioned within the pedicles and in the VBS under fluoroscopic guidance, with no screw loosening observed at follow-up. The screws implanted in the SAIF technique are not connected to external fixation bars; thus, there are no strong stress forces on the screws that can predispose to screw loosening.

\section{SAIF Stabilization Efficacy}

The main aim of the SAIF procedure is VB reconstruction to restore axial load-bearing capability while avoid- 

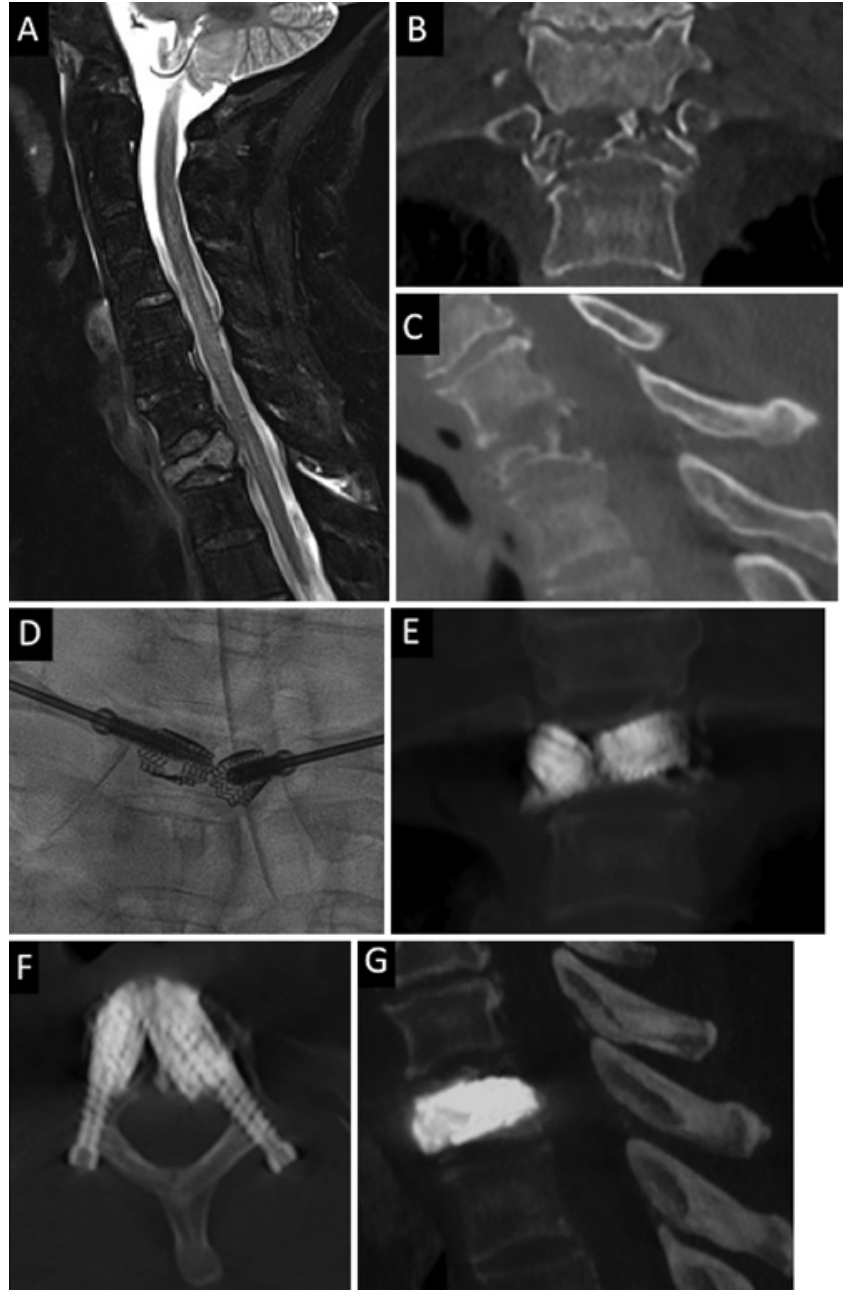

FIG. 4. Images of a breast cancer metastatic $T 1$ fracture in a 56-year-old patient. MRI (A) and CT (B-C) showed the severe VB lytic destruction and collapse. Long-segment posterior instrumentation would have limited the patient's mobility, and a T1 corpectomy would have posed significant surgical challenges. The patient could be treated with a percutaneous, minimally invasive, stand-alone SAIF approach (D). Postprocedure CT $(E-G)$ displayed the optimal VB reconstruction by stents augmented with bone cement, anchored to the posterior elements by transpedicular fenestrated screws.

ing more invasive traditional surgery in this compromised cohort. A previously published qualitative score based on postprocedure plain radiographs and CT images was used to assess the reconstruction: ${ }^{9}$ the construct was judged satisfactory when appropriate placement of the devices, VBS expansion, and cement filling restored VB height and achieved reconstruction of the destroyed VB, appearing as a $360^{\circ}$ nonfusion internal fixation of the affected VB. The reconstruction was judged satisfactory (good or excellent) in $94.5 \%$ of cases by the two raters, with high interrater agreement.

Follow-up, ranging from 1 to 30 months, was available for 30 treated levels. At follow-up, the implants were stable with no VBS mobilization in 29/30 cases. The screw anchoring may represent a means of avoiding VBS mobilization in conjunction with other technical measures, such as a PMMA bridge, cement interdigitation, and optimized implant(s) positioning. In the late follow-up CT studies ( $\geq$ 6 months postprocedure), formation of a new cortical bone shell was noted around the construct, and in some cases intervertebral osseous fusion was observed (Fig. 3), suggesting the achievement of stability. Clinical and imaging follow-up was limited by patient death or loss to followup, but is nevertheless representative in such a cohort with advanced metastatic disease.

In a complex case treated with bilateral VBSs and a unilateral-screw SAIF for lung cancer metastatic thoracic EO, mobilization of the non-screw-anchored VBS occurred. The ex post facto case analysis revealed a series of clinical and technical errors likely contributing to the occurrence of this unfortunate complication. The patient had unrecognized osteomyelitis at the metastatic vertebral level that worsened and evolved into an epidural abscess. Moreover, the presence of osteolysis involving one pedicle suggested unilateral screw fixation on the intact side, but a "kissing configuration" of the VBSs was made impossible by a sclerotic spur within the otherwise lytic VB, which eventually prevented the desired creation of a PMMA bridge between the two VBSs. Interestingly, evolving osteomyelitis likely caused mobilization of the nonanchored VBS, while the screw-anchored VBS remained in place.

The majority of patients with EO experience mechanical pain. While pain palliation is an indication for treatment, it did not represent a formal endpoint of the present study. Our rationale was straightforward. Thousands of studies, including randomized controlled trials in which patients were randomized against conservative therapy in both osteoporotic and neoplastic fractures, have demonstrated meaningful pain improvement with augmentation. ${ }^{4,27,42}$ Even sham studies that fail to demonstrate the expected differential benefit have included augmentation patients whose pain dramatically improves after the procedure. ${ }^{17}$ In our cohort with EO and diffuse metastatic disease, concomitant VA was deemed necessary at adjacent or distant levels in 22/35 patients (61 levels; Fig. 3), and these patients concurrently received different regimens of radiotherapy, chemotherapy, and supportive care (including steroids and analgesic drugs), ${ }^{12}$ including for metastatic deposits outside of the skeleton, e.g., the viscera. Despite these confounding factors, in a retrospective review of the medical records, an experienced medical oncologist (V.E.) assessed pain amelioration potentially attributable to the SAIF procedure in 19/35 patients (with no new or changed therapeutic regimen). In 4/35 patients, pain amelioration was attributable to the procedure in combination with chemo- or radiotherapy, while in $7 / 35$ patients no significant pain amelioration was noted after the procedure. In 1 case pain was not evaluable because the patient had died 2 days after the procedure because of a pulmonary embolism unrelated to PMMA migration. The remaining 4/35 patients had no definite preprocedure pain clearly attributable to the vertebral target lesion. In this study we utilized a clinical oncological evaluation rather than a formal visual ana$\log$ scale. The outcomes illustrated symptomatic improvement and are comparable to those previously reported with the use of VBS in extensive VB lytic lesions. ${ }^{9}$

The SAIF procedure allows patients to recover quickly 

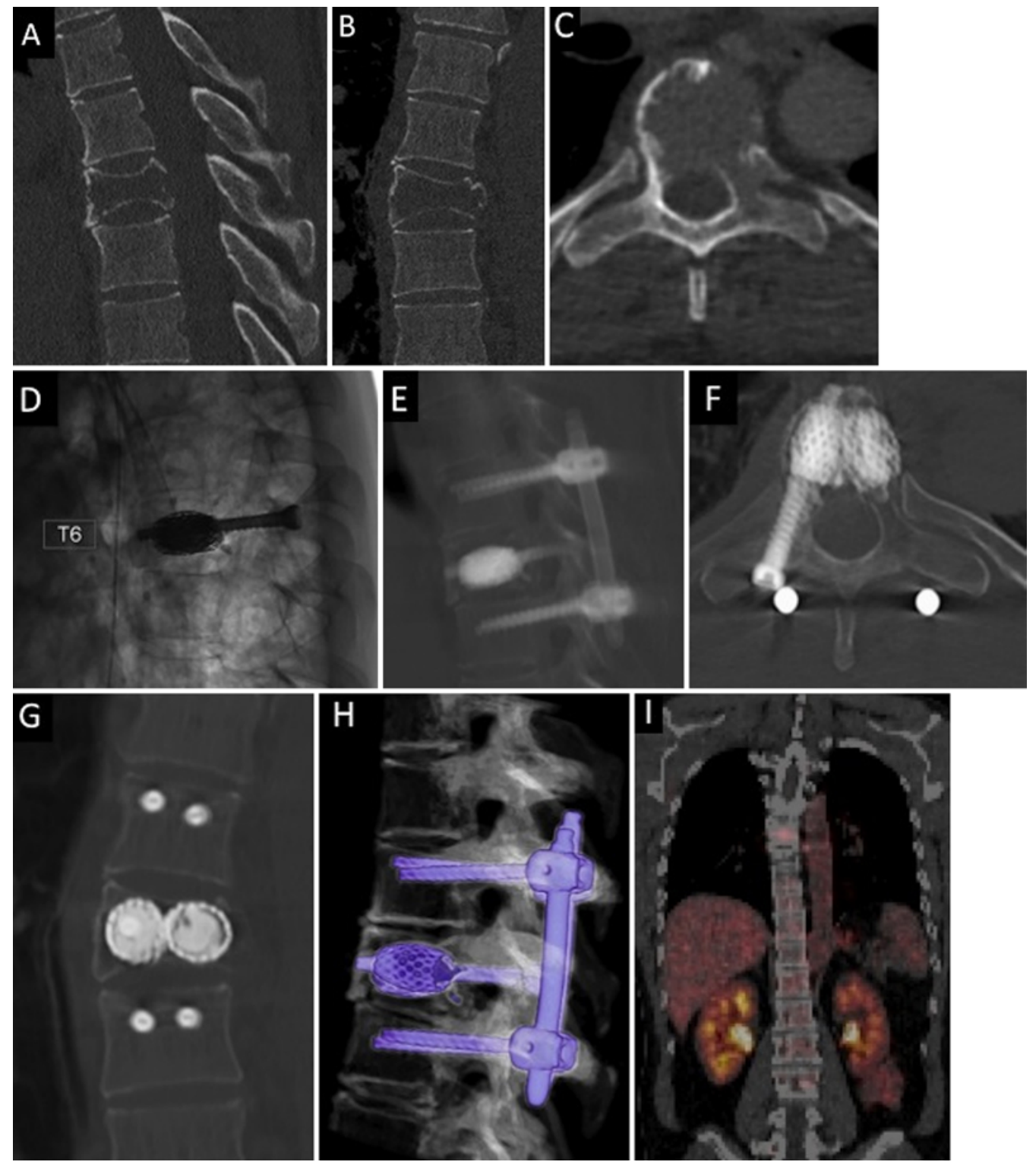

FIG. 5. Images of a solitary plasmocytoma of T6 in a very tall and fit 52-year-old patient. Multiplanar CT images (A-C) showed EO of the VB also involving the left pedicle and the impending asymmetrical fracture causing scoliotic deformity in the frontal plane (B). The SAIF technique with bilateral stents and a unilateral screw was performed to reconstruct the VB (D) and avoid corpectomy. In the same anesthesia period, percutaneous short-segment dorsal instrumentation was added, as shown by postprocedure CT $(\mathrm{E}-\mathrm{H})$, to provide further stability given the patient's body habitus. PET-CT follow-up at 12 months (I), following chemotherapy, showed stable spinal alignment and no signs of residual or recurrent disease. Figure is available in color online only.

as compared to their recovery following open surgery. Its less invasive nature facilitates the start or resumption of radiotherapy or chemotherapy soon after the procedure. Moreover, the proposed SAIF technique offers the advantage of immediate stabilization of the VB, while radiotherapy alone cannot ensure stabilization. Indeed, stand-alone radiotherapy can be followed by a phase of increased vertebral fragility and fracture risk. ${ }^{23,37}$

Furthermore, patients with mechanical pain attributable to metastatic destruction or collapse of the VB with resulting intervertebral instability are nonresponders to radiotherapy alone. These patients generally require some form of stabilization procedure to address their mechanical in- stability. ${ }^{29}$ Thus, we believe SAIF to have a complementary role to treatment with radiation and/or chemotherapy to achieve immediate VB reconstruction and stabilization, rapid pain relief, and local disease control. ${ }^{23}$

The clinical protocol utilized by practitioners involved in our multidisciplinary spine tumor board is to consider stabilization via SAIF when a patient has a VB affected by EO and would have difficulty recuperating in a timely fashion from more traditional operative approaches. In fact, EO lesions commonly demonstrate wide cortical boundary erosion and bone comminution, advanced vertebral collapse, and an epidural mass, which all represent at least relative contraindications to more standard VA 
procedures. In these patients SAIF could represent a minimally invasive option when anterior column reconstructive surgery is contraindicated or not desired. Finally, in the presence of lytic involvement of the posterior elements, signs of gross instability such as fracture-related kyphotic or scoliotic deformity, or the highest SINSs or when decompressive laminectomy is indicated, SAIF can be combined with a posterior surgical approach, as was done in $5 / 35$ patients in this series.

\section{Complications}

The majority of complications were technical and asymptomatic. Two patients $(5.7 \%)$ reported a clinical complication secondary to an epidural leak, including an L2 sensory and motor deficit necessitating surgical posterior decompression, with no permanent deficit, and transient self-limiting L5 radicular pain for which no treatment was required. Two previous studies have reported a similar rate of epidural cement leakage, either during VA of neoplastic lytic lesions with a dehiscent posterior wall $(14.2 \%)^{10}$ or during VBS augmentation in EO $(12.2 \%) .{ }^{9}$ No other patients experienced worsening of their neurological status postprocedure. One patient with metastatic lung cancer died on postoperative day 1 as the result of a nonPMMA-related pulmonary embolism, which can be considered a non-SAIF-specific perioperative complication.

One nonanchored VBS mobilized at the 1-month follow-up and required surgical correction. This situation was likely related to underlying osteomyelitis. We recommend bilateral screw fixation of the VBSs; whenever this is not possible, a "kissing configuration" of the VBSs should be obtained to ensure the creation of a PMMA bridge between the two VBSs. Similarly, we do not recommend the SAIF technique if both pedicles are involved by the osteolysis and do not ensure safe osseous anchoring via the screw(s).

This SAIF series, in which a total of 2/36 levels required repeat surgery, compares favorably to a recent large retrospective series of posterior instrumentation, separation surgery, and anterior column cement reconstruction, ${ }^{2}$ which reported an $18 \%$ overall rate of multiple surgeries due to wound complications, CSF leakage, tumor recurrence, hardware failure, and other causes.

\section{Study Limitations}

The principal limitations of this study are attributable to its real-world design, relatively small sample size, and the absence of a systematic clinicoradiological follow-up. Moreover, this study lacked a control group; however, as previously mentioned, the multidisciplinary team considered the patients to be poor candidates either for VA because of their EO lesion features or for anterior column surgery because of their clinical conditions. The VB reconstruction scale that we utilized is qualitative but did have optimal interrater agreement between raters from two distinct disciplines.

The strength of this study relates to the prospectively established database utilized, with all treated cases managed and followed up based on an established multidisciplinary clinical practice. We believe that this study may represent a preliminary step to support SAIF, a promising new technique, and that a larger study may provide stronger information upon which to base this minimally invasive approach of stabilization in patients with extensive lytic VB lesions. In addition, FEM analysis data provide a supportive biomechanical rationale for use of the technique in $\mathrm{EO}$ cases. ${ }^{31}$

\section{Conclusions}

The SAIF technique allows $360^{\circ}$ nonfusion internal fixation to stabilize the VB in patients with extensive lytic lesions. SAIF appears to be an effective stabilization procedure with durable results at follow-up, and while there were transient and permanent complications, the population targeted for this procedure is inherently challenging. Therefore, SAIF may represent an alternative option to a more invasive corpectomy, either as a stand-alone intervention or in combination with a posterior surgical approach of decompression and stabilization.

\section{References}

1. Altaf F, Weber M, Dea N, Boriani S, Ames C, Williams R, et al: Evidence-based review and survey of expert opinion of reconstruction of metastatic spine tumors. Spine (Phila Pa 1976) 41 (Suppl 20):S254-S261, 2016

2. Amankulor NM, Xu R, Iorgulescu JB, Chapman T, Reiner AS, Riedel E, et al: The incidence and patterns of hardware failure after separation surgery in patients with spinal metastatic tumors. Spine J 14:1850-1859, 2014

3. Anselmetti GC, Zoarski G, Manca A, Masala S, Eminefendic $\mathrm{H}$, Russo F, et al: Percutaneous vertebroplasty and bone cement leakage: clinical experience with a new high-viscosity bone cement and delivery system for vertebral augmentation in benign and malignant compression fractures. Cardiovasc Intervent Radiol 31:937-947, 2008

4. Berenson J, Pflugmacher R, Jarzem P, Zonder J, Schechtman K, Tillman JB, et al: Balloon kyphoplasty versus non-surgical fracture management for treatment of painful vertebral body compression fractures in patients with cancer: a multicentre, randomised controlled trial. Lancet Oncol 12:225-235, 2011

5. Bilsky MH, Laufer I, Fourney DR, Groff M, Schmidt MH, Varga PP, et al: Reliability analysis of the epidural spinal cord compression scale. J Neurosurg Spine 13:324-328, 2010

6. Boriani S, Gasbarrini A, Bandiera S, Ghermandi R, Lador R: En bloc resections in the spine: the experience of 220 patients during 25 years. World Neurosurg 98:217-229, 2017

7. Cazzato RL, Koch G, Buy X, Ramamurthy N, Tsoumakidou G, Caudrelier J, et al: Percutaneous image-guided screw fixation of bone lesions in cancer patients: double-centre analysis of outcomes including local evolution of the treated focus. Cardiovasc Intervent Radiol 39:1455-1463, 2016

8. Cianfoni A, Distefano D, Isalberti M, Reinert M, Scarone P, Kuhlen D, et al: Stent-screw-assisted internal fixation: the SAIF technique to augment severe osteoporotic and neoplastic vertebral body fractures. J Neurointerv Surg 11:603609, 2019

9. Cianfoni A, Distefano D, Pravatà E, Espeli V, Pesce G, Mordasini $P$, et al: Vertebral body stent augmentation to reconstruct the anterior column in neoplastic extreme osteolysis. J Neurointerv Surg 11:313-318, 2019

10. Cianfoni A, Raz E, Mauri S, Di Lascio S, Reinert M, Pesce $\mathrm{G}$, et al: Vertebral augmentation for neoplastic lesions with posterior wall erosion and epidural mass. AJNR Am J Neuroradiol 36:210-218, 2015 
11. Cohen J: A coefficient of agreement for nominal scales. Educ Psychol Meas 20:37-46, 1960

12. Dawson EG, Kanim LEA, Sra P, Dorey FJ, Goldstein TB, Delamarter RB, et al: Low back pain recollection versus concurrent accounts: outcomes analysis. Spine (Phila Pa 1976) 27:984-994, 2002

13. Deschamps F, de Baere T, Hakime A, Pearson E, Farouil G, Teriitehau C, et al: Percutaneous osteosynthesis in the pelvis in cancer patients. Eur Radiol 26:1631-1639, 2016

14. Deschamps F, Farouil G, Hakime A, Teriitehau C, Barah A, de Baere T: Percutaneous stabilization of impending pathological fracture of the proximal femur. Cardiovasc Intervent Radiol 35:1428-1432, 2012

15. Diel P, Röder C, Perler G, Vordemvenne T, Scholz M, Kandziora F, et al: Radiographic and safety details of vertebral body stenting: results from a multicenter chart review. BMC Musculoskelet Disord 14:233, 2013

16. Fang T, Dong J, Zhou X, McGuire RA Jr, Li X: Comparison of mini-open anterior corpectomy and posterior total en bloc spondylectomy for solitary metastases of the thoracolumbar spine. J Neurosurg Spine 17:271-279, 2012

17. Firanescu C, Lohle PN, de Vries J, Klazen CA, Juttmann JR, Clark W, et al: A randomised sham controlled trial of vertebroplasty for painful acute osteoporotic vertebral fractures (VERTOS IV). Trials 12:93, 2011

18. Fourney DR, Frangou EM, Ryken TC, Dipaola CP, Shaffrey CI, Berven SH, et al: Spinal instability neoplastic score: an analysis of reliability and validity from the Spine Oncology Study Group. J Clin Oncol 29:3072-3077, 2011

19. Fourney DR, Schomer DF, Nader R, Chlan-Fourney J, Suki D, Ahrar K, et al: Percutaneous vertebroplasty and kyphoplasty for painful vertebral body fractures in cancer patients. J Neurosurg 98 (1 Suppl):21-30, 2003

20. Garnon J, Koch G, Ramamurthy N, Caudrelier J, Rao P, Tsoumakidou G, et al: Percutaneous CT and fluoroscopyguided screw fixation of pathological fractures in the shoulder girdle: technical report of 3 cases. Cardiovasc Intervent Radiol 39:1332-1338, 2016

21. Gonschorek O, Hauck S, Weiß T, Bühren V: Percutaneous vertebral augmentation in fragility fractures-indications and limitations. Eur J Trauma Emerg Surg 43:9-17, 2017

22. Gu Y, Dong J, Jiang X, Wang Y: Minimally invasive pedicle screws fixation and percutaneous vertebroplasty for the surgical treatment of thoracic metastatic tumors with neurologic compression. Spine (Phila Pa 1976) 41 (Suppl 19):B14B22, 2016

23. Hirsch AE, Jha RM, Yoo AJ, Saxena A, Ozonoff A, Growney MJ, et al: The use of vertebral augmentation and external beam radiation therapy in the multimodal management of malignant vertebral compression fractures. Pain Physician 14:447-458, 2011

24. Hirsch JA, Chandra RV, Pampati V, Barr JD, Brook AL, Manchikanti L: Analysis of vertebral augmentation practice patterns: a 2016 update. J Neurointerv Surg 8:1299-1304, 2016

25. Ikard RW: Methods and complications of anterior exposure of the thoracic and lumbar spine. Arch Surg 141:1025-1034, 2006

26. Jha RM, Hirsch AE, Yoo AJ, Ozonoff A, Growney M, Hirsch JA: Palliation of compression fractures in cancer patients by vertebral augmentation: a retrospective analysis. J Neurointerv Surg 2:221-228, 2010

27. Klazen CAH, Lohle PNM, de Vries J, Jansen FH, Tielbeek $\mathrm{AV}$, Blonk MC, et al: Vertebroplasty versus conservative treatment in acute osteoporotic vertebral compression fractures (Vertos II): an open-label randomised trial. Lancet 376:1085-1092, 2010

28. Klezl Z, Majeed H, Bommireddy R, John J: Early results after vertebral body stenting for fractures of the anterior column of the thoracolumbar spine. Injury 42:1038-1042, 2011
29. Krishnaney AA, Steinmetz MP, Benzel EC: Biomechanics of metastatic spine cancer. Neurosurg Clin N Am 15:375-380, 2004

30. Kwan MK, Lee CK, Chan CYW: Minimally invasive spinal stabilization using fluoroscopic-guided percutaneous screws as a form of palliative surgery in patients with spinal metastasis. Asian Spine J 10:99-110, 2016

31. La Barbera L, Cianfoni A, Ferrari A, Distefano D, Bonaldi G, Villa T: Stent screw-assisted internal fixation (SAIF) of severe lytic spinal metastases: a comparative finite element analysis of the SAIF technique. World Neurosurg 128:e370-e377, 2019

32. Landis JR, Koch GG: The measurement of observer agreement for categorical data. Biometrics 33:159-174, 1977

33. Laufer I, Bilsky MH: Advances in the treatment of metastatic spine tumors: the future is not what it used to be. J Neurosurg Spine 30:299-307, 2019

34. Laufer I, Sciubba DM, Madera M, Bydon A, Witham TJ, Gokaslan ZL, et al: Surgical management of metastatic spinal tumors. Cancer Contr 19:122-128, 2012

35. Piechowiak EI, Isalberti M, Pileggi M, Distefano D, Hirsch JA, Cianfoni A: Mechanical cavity creation with curettage and vacuum suction (Q-VAC) in lytic vertebral body lesions with posterior wall dehiscence and epidural mass before cement augmentation. Medicina (Kaunas) 55:633, 2019

36. Pusceddu C, Fancellu A, Ballicu N, Fele RM, Sotgia B, Melis L: CT-guided percutaneous screw fixation plus cementoplasty in the treatment of painful bone metastases with fractures or a high risk of pathological fracture. Skeletal Radiol 46:539545,2017

37. Rose PS, Laufer I, Boland PJ, Hanover A, Bilsky MH, Yamada J, et al: Risk of fracture after single fraction image-guided intensity-modulated radiation therapy to spinal metastases. J Clin Oncol 27:5075-5079, 2009

38. Sciubba DM, Petteys RJ, Dekutoski MB, Fisher CG, Fehlings MG, Ondra SL, et al: Diagnosis and management of metastatic spine disease. A review. J Neurosurg Spine 13:94108,2010

39. Steinmetz MP, Mekhail A, Benzel EC: Management of metastatic tumors of the spine: strategies and operative indications. Neurosurg Focus 11(6):e2, 2001

40. Thaler M, Lechner R, Nogler M, Gstöttner M, Bach C: Surgical procedure and initial radiographic results of a new augmentation technique for vertebral compression fractures. Eur Spine J 22:1608-1616, 2013

41. Tomita K, Kawahara N, Kobayashi T, Yoshida A, Murakami H, Akamaru T: Surgical strategy for spinal metastases. Spine (Phila Pa 1976) 26:298-306, 2001

42. Wardlaw D, Cummings SR, Van Meirhaeghe J, Bastian L, Tillman JB, Ranstam J, et al: Efficacy and safety of balloon kyphoplasty compared with non-surgical care for vertebral compression fracture (FREE): a randomised controlled trial. Lancet 373:1016-1024, 2009

43. Werner CML, Osterhoff G, Schlickeiser J, Jenni R, Wanner GA, Ossendorf C, et al: Vertebral body stenting versus kyphoplasty for the treatment of osteoporotic vertebral compression fractures: a randomized trial. J Bone Joint Surg Am 95:577-584, 2013

44. Yang Z, Yang Y, Zhang Y, Zhang Z, Chen Y, Shen Y, et al: Minimal access versus open spinal surgery in treating painful spine metastasis: a systematic review. World J Surg Oncol 13:68, 2015

\section{Disclosures}

Dr. Hirsch is a consultant for Medtronic, Relievent, and Cerenovus and holds positions with Data and Safety Monitoring Board and Data Monitoring Committee. 


\section{Author Contributions}

Conception and design: all authors. Acquisition of data: Distefano, Cianfoni, Scarone, Espeli. Analysis and interpretation of data: Distefano, Cianfoni, Scarone, Pesce, Espeli, La Barbera, Villa. Drafting the article: all authors. Critically revising the article: Pesce, Bonaldi, Hirsch. Statistical analysis: Distefano. Study supervision: Cianfoni, Villa, Bonaldi, Hirsch.

\section{Supplemental Information}

\section{Previous Presentations}

Portions of the data included in this study have been presented as an oral presentation at the Spine Interventional Neuroradiology Section at the 57th Annual Congress of the American Society of Neuroradiology held in Boston, Massachusetts, in May 2019.

\section{Correspondence}

Daniela Distefano: Neurocenter of Southern Switzerland, Ospedale Regionale di Lugano, Lugano, Switzerland. daniela. distefano@eoc.ch. 